Please quote as: Gebauer, L.; Söllner, M. \& Leimeister, J. M. (2016): Vertrauensunterstützende Mehrwertdienste - Ein systematisches Vorgehen zur Förderung von Vertrauen im Cloud-Computing-Umfeld. In: Cloud-Services aus der Geschäftsperspektive. Hrsg./Editors: Krcmar, H.; Leimeister, J. M.; Roßnagel, A. \& Sunyaev, A. Verlag/Publisher: Springer Gabler, Wiesbaden. Erscheinungsjahr/Year: 2016. Seiten/Pages: 171-185. 


\title{
13 Vertrauensunterstützende Mehrwertdienste - Ein systematisches Vorgehen zur Förderung von Vertrauen im Cloud-Computing-Umfeld
}

\author{
L. Gebauer, M. Söllner, J.M. Leimeister
}

\subsection{Prozess zur systematischen Vertrauensunterstützung}

In diesem Kapitel wird ein Prozess zur Entwicklung vertrauensunterstützender Mehrwertdienste erarbeitet, um mit den daraus abgeleiteten Lösungen den Vertrauensproblematiken im Cloud-Computing-Umfeld (siehe Kapitel 6) zu begegnen. Dazu wurde die Methode zur Entwicklung vertrauensunterstützender Komponenten für sozio-technische ubiquitäre Systeme nach Söllner et al. (2012a) auf den Cloud-Computing-Kontext übertragen und weiterentwickelt.

Der Prozess zur systematischen Vertrauensunterstützung umfasst fünf aufeinander folgende Schritte (Abbildung 1) und soll Cloud-Computing-Anbieter befähigen ihre Cloud-Computing-Dienste vertrauenswürdiger zu gestalten. Jeder einzelne Schritt wird durch eine Leitfrage angeleitet, um so die Erarbeitung der einzelnen Entwicklungsaktivitäten zu unterstützen. Der Prozess zielt darauf ab, Lösungen in Form von vertrauensunterstützenden Mehrwertdiensten für den ausgewählten Cloud-Computing-Dienst abzuleiten. In den nachfolgenden Abschnitten wird der Prozess zur systematischen Vertrauensunterstützung erklärt und allgemein für Cloud-Computing durchlaufen, jedoch ohne Bezugnahme auf einen konkreten Dienst. Die exemplarischen Ergebnisse können von den Cloud-Computing-Anbietern als Grundlage verwendet werden, um eigene, individuelle Lösungen zu entwickeln. Dennoch empfiehlt es sich, eine kritische Überprüfung hinsichtlich der Übertragbarkeit auf den ausgewählten Cloud-Computing-Dienst durchzuführen. Es kann nicht ausgeschlossen werden, dass bestimmte Aspekte für den ausgewählten Cloud-Computing-Dienst keine Relevanz haben oder wiederum wichtige Aspekte noch nicht berücksichtigt wurden. 


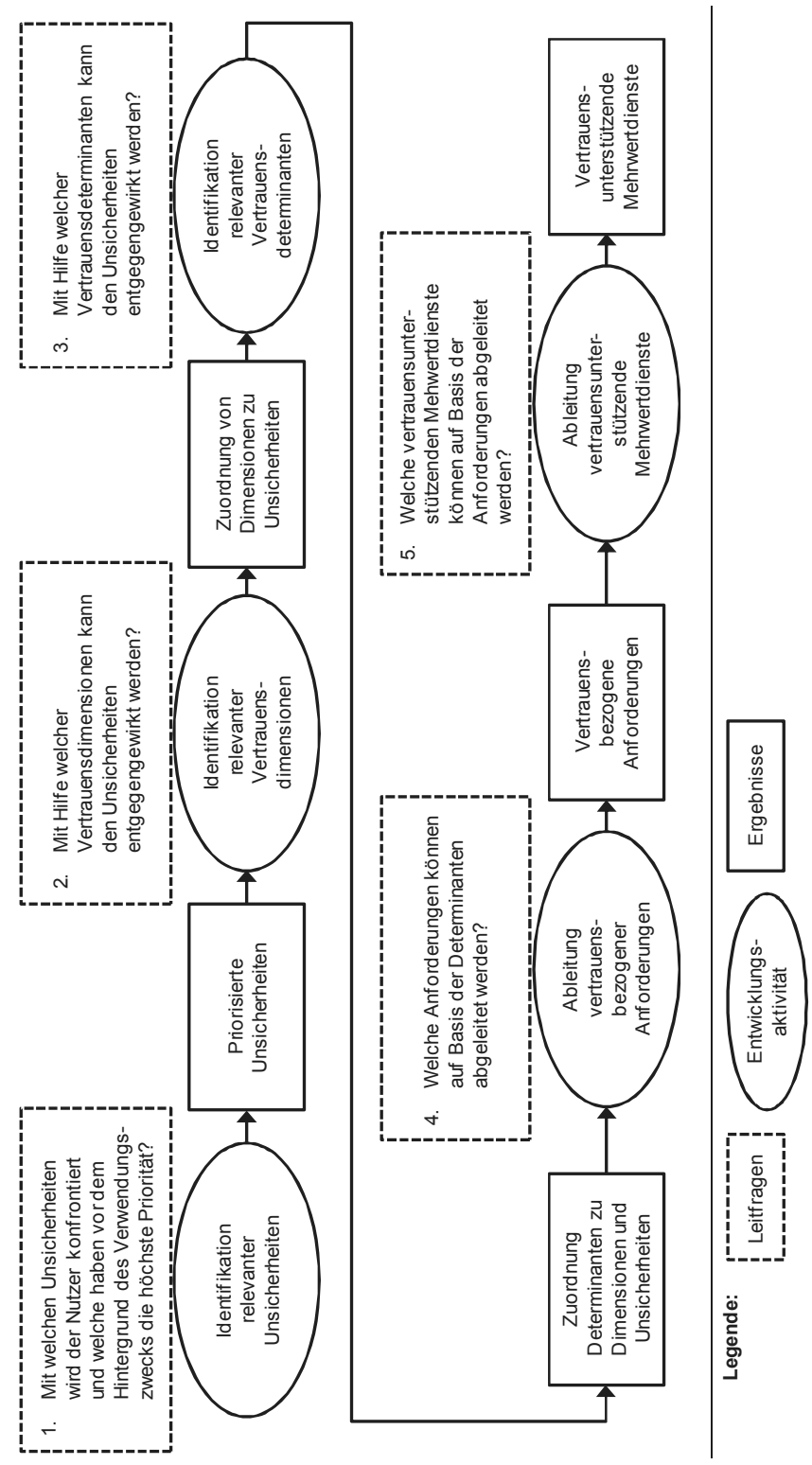

Abbildung 1: Prozess zur systematischen Vertrauensunterstützung für Cloudbasierte Dienstleistungen (In Anlehnung an Söllner et al. 2012a). 
Im ersten Schritt des Prozesses zur systematischen Vertrauensunterstützung werden die Unsicherheiten bzw. die Nutzungshemmnisse der (potenziellen) Cloud-Computing-Nutzer identifiziert und analysiert. Im zweiten und dritten Schritt werden die relevanten Vertrauensdimensionen und -determinanten bestimmt, mit denen den identifizierten Unsicherheiten und Hemmnissen entgegengewirkt werden kann. Im vierten und fünften Schritt werden aufbauend auf den Ergebnissen vertrauensbezogene Anforderungen sowie vertrauensunterstützende Lösungen bzw. Mehrwertdienste erarbeitet.

\subsection{Schritt 1: Identifikation relevanter Unsicherheiten}

Im ersten Schritt erfolgt die Entwicklungsaktivität „Identifikation relevanter Unsicherheiten“. Im Zuge derer wird folgende Leitfrage beantwortet: „Mit welchen Unsicherheiten wird der Nutzer konfrontiert und welche haben vor dem Hintergrund des Verwendungszwecks die höchste Priorität?“". Zur exemplarischen Beantwortung dieser Frage werden die folgenden Kategorien von Nutzungshemmnissen genannt (siehe Kapitel 6), wobei deren Gültigkeit in Bezug auf den zu untersuchenden Cloud-Computing-Dienst überprüft und deren Relevanz im Hinblick auf den Verwendungszweck priorisiert werden sollte:

- Diskontinuität/ Ausfall des Dienstes

- Systemangriffe

- Netzwerkstörungen

- begrenzte Skalierbarkeit

- Zeit- und kostenintensive Datenübertragung

- mangelnde Datenintegrität

- Abhängigkeit vom Anbieter

- Abhängigkeit von anderen Nutzern

- mangelnde Kontrollmöglichkeiten

- mangelnde Reliabilität 
- mangelnde rechtliche Standards

- Gefährdung interner IT

Workshops mit einem Anbieterunternehmen von Cloud-Computing-Diensten zeigen, dass es empfehlenswert ist, die (potenziellen) Kunden direkt nach deren Unsicherheiten und Bedenken gegenüber der Nutzung des Cloud-Computing-Dienstes zu befragen. In den Workshops ergaben sich beispielhaft die folgenden Unsicherheiten, Bedenken oder Fragen:

- Sicherstellung der Verfügbarkeit fraglich, z.B. aufgrund mangelnder Internetanbindung in verschiedenen Regionen

- Gewährleistung einer sicheren Verbindung zwischen internen und externen Mitarbeitern

- Bereitstellung einer maßgeschneiderten Kundenlösung fraglich

- Was ist günstiger: Service mieten oder eigenes Rechenzentrum betreiben?

- Schnittstellenprobleme durch unterschiedliche Software/ Betriebssysteme

- Sensible/ personenbezogene Daten: Was darf in die Cloud und was nicht?

- Unübersichtlichkeit am Markt: Wie finde ich bei den vielen Anbietern den richtigen Anbieter?

- Unübersichtlichkeit der Leistungsangebote: Wie finde ich den CloudDienst, der zu mir passt?

Abschließend sollten die identifizierten Unsicherheiten in eine Rangfolge gebracht werden, um herauszustellen, welche Unsicherheiten vor dem Hintergrund des Verwendungszwecks des Cloud-Computing-Dienstes bei den (potenziellen) Kunden am stärksten ausgeprägt sind. 


\subsection{Schritt 2: Identifikation relevanter Vertrauensdimensionen}

Im zweiten Schritt wird der Leitfrage nachgegangen: „Mit Hilfe welcher Vertrauensdimensionen kann den Unsicherheiten entgegengewirkt werden?", um die Entwicklungsaktivität „Identifikation relevanter Vertrauensdimensionen " zu adressieren. Bei der Beantwortung der Leitfrage wird zwischen dem interpersonellen Vertrauen, also dem Vertrauen in den Anbieter von CloudComputing-Diensten, und dem Systemvertrauen, also dem Vertrauen in die Cloud-Computing-Technik an sich, unterschieden. Forschungsarbeiten zeigen, dass sich diese beiden Komponenten von Vertrauen aus mehreren Dimensionen ergeben, die wiederum von unterschiedlichen Determinanten beeinflusst werden (Abbildung 2).

Vertrauen in den Anbieter setzt sich gemäß der Forschung (Söllner et al. 2013; Mayer et al. 1995) aus drei Dimensionen zusammen (vgl. Abbildung 2):

- Fähigkeit (im englischen Original: Ability),

- Wohlwollen (im englischen Original: Benevolence) und

- Integrität (im englischen Original: Integrity).

Die Dimension Fähigkeit beschreibt die Einschätzung des Vertrauensgebers, also des (potenziellen) Cloud-Computing-Nutzers, inwieweit dieser den Vertrauensnehmer, also den Cloud-Computing-Anbieter, für fähig erachtet, bei der Zielerreichung behilflich zu sein (Söllner et al. 2012a). Die Dimension Wohlwollen ist die Einschätzung des (potenziellen) Cloud-ComputingNutzers, inwieweit der Cloud-Computing-Anbieter bereit ist, die Interessen des Nutzers zu berücksichtigen und zu wahren (Söllner et al. 2012a). Integrität ist die Einschätzung des (potenziellen) Cloud-Computing-Nutzers, inwieweit sich der Cloud-Computing-Anbieter an gewissen Prinzipien und Normen orientiert, die der Nutzer für gut erachtet (Söllner et al. 2012a). 


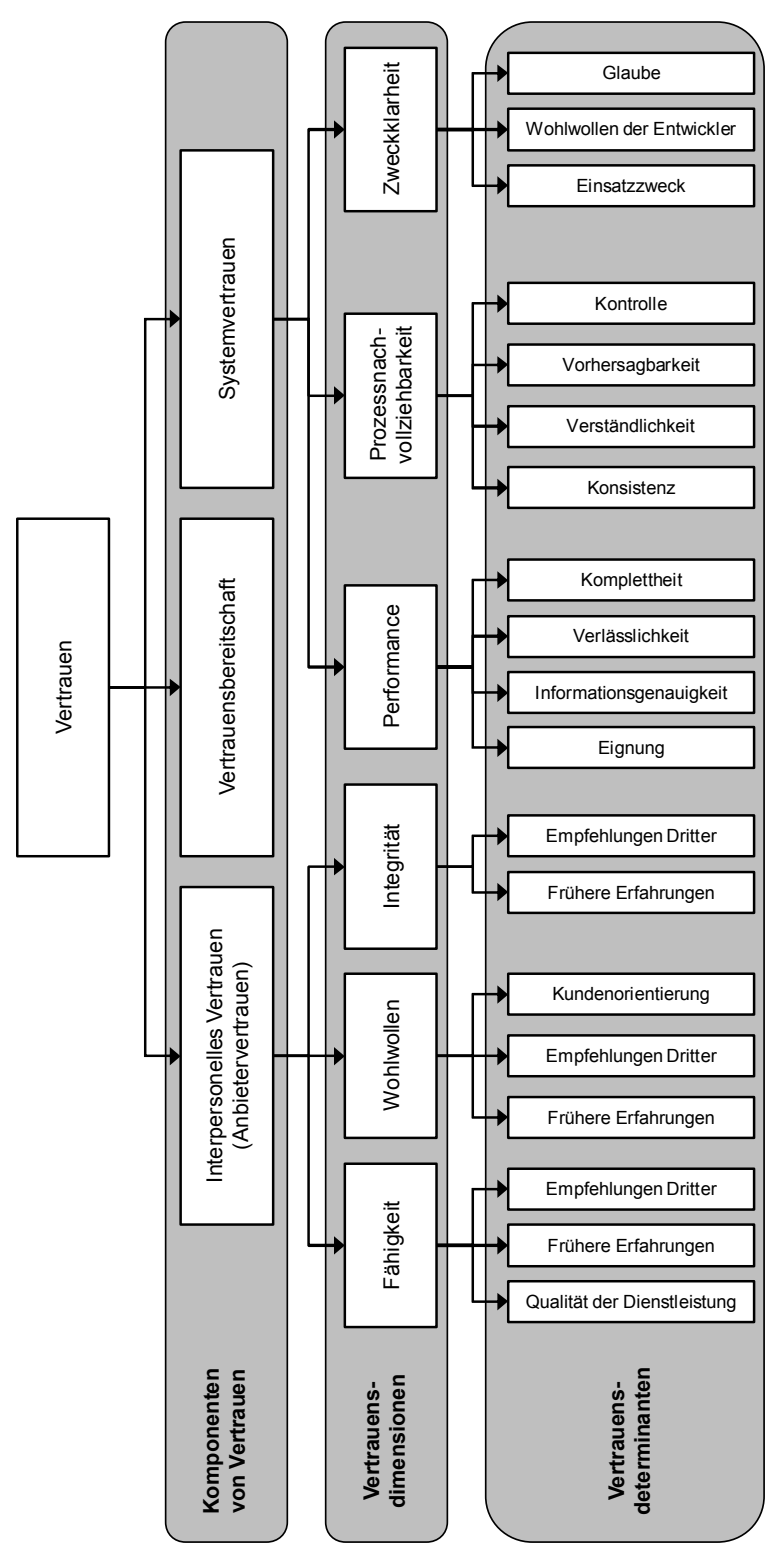

Abbildung 2: Überblick Vertrauen im Cloud-Computing-Umfeld (Quelle: Eigene Darstellung). 
Das Systemvertrauen ergibt sich gemäß verschiedener Forschungsarbeiten (z. B. Söllner et al. 2012b; Lee/See 2004) aus der Einschätzung folgender Dimensionen (vgl. Abbildung 2):

- Performance (im englischen Original: Performance),

- Prozessnachvollziehbarkeit (im englischen Original: Process) und

- Zweckklarheit (im englischen Original: Purpose).

Die Dimension Performance umfasst „die Einschätzungen des Nutzers dahingehend, wie gut ein System dazu in der Lage ist, ihn bei der Erreichung seiner Ziele zu unterstützen" (Söllner et al. 2012a, S. 117). Prozessnachvollziehbarkeit bedeutet die Nutzereinschätzung, ,wie das System funktioniert und welche grundlegenden Prinzipien beachtet werden" (Söllner et al. 2012a, S. 117). Zweckklarheit betrachtet weniger die Eigenschaften des Systems, sondern die Beurteilung aus der Sicht des Nutzers, zu welchem Zweck das System entwickelt wurde. Deckt sich dieser Zweck mit dem Ziel des Nutzers, ,so entsteht das Gefühl, dass das System die Anliegen des Nutzers in den Vordergrund stellt und beachtet" (Söllner et al. 2012a, S. 117).

Auch wenn Forschungsarbeiten zeigen, dass diese Dimensionen Vertrauen in Informationstechnologien und deren Anbieter ausmachen, sollten die Cloud-Computing-Anbieter kritisch überprüfen, ob diese Dimensionen auch für den betrachteten Cloud-Computing-Dienst relevant sind. Zudem sind diese Dimensionen noch sehr abstrakt und lassen sich nicht direkt zu vertrauensbezogene Anforderungen ableiten (Söllner et al. 2012a), weshalb im nächsten Schritt konkrete Vertrauensdeterminanten erarbeitet werden.

\subsection{Schritt 3: Identifikation relevanter Vertrauensdeterminanten}

Im dritten Schritt wird die Entwicklungsaktivität „Identifikation relevanter Vertrauensdeterminanten“ bearbeitet und die Leitfrage beantwortet: „Mit Hilfe welcher Vertrauensdeterminanten kann den Unsicherheiten entgegengewirkt werden?".

Die Forschung liefert bisher wenige Erkenntnisse, welche konkreten Determinanten das interpersonelle Vertrauen in den IT-Anbieter beeinflussen. 
Um dennoch exemplarisch Vertrauensdeterminanten vorzugeben, wurden Interviews mit Cloud-Computing-Experten (siehe Kapitel 22) durchgeführt. Gemäß der Auswertung der Experteninterviews (Buch/Gebauer 2013; Buch et al. 2014), beeinflussen die folgenden Determinanten das Vertrauen in den Cloud-Computing-Anbieter (vgl. Abbildung 2):

- Qualität der Dienstleistung (umfasst Aspekte, die herangezogen werden, um Rückschlüsse auf die Qualität der Dienstleistung und dessen Anbieter zu ziehen, bspw. Pünktlichkeit, die Verfügbarkeit, die Zuverlässigkeit),

- frühere Erfahrungen mit Dienstleistern (umfasst alle früheren Erfahrungen, die der potenzielle Kunde zuvor schon selbst mit dem Dienstleister gemacht hat),

- Empfehlungen Dritter (umfasst alle persönlichen Empfehlungen, die der potenzielle Kunde von Dritten erhalten hat, wie zum Beispiel von Kollegen, Freunden oder von eingestellten Beratern) und

- Kundenorientierung des Dienstleisters (umfasst, inwieweit der Dienstleister sich auf seine Kunden einstellt und auf dessen Wünsche eingeht) (Buch et al. 2014).

Die Zuordnung der identifizierten Determinanten anhand der Experteninterviews zu den Dimensionen erfolgte rein deskriptiv. Die statistischen $\mathrm{Zu}$ sammenhänge sowie die Vollständigkeit sollten in weiteren Studien empirisch überprüft werden.

Für das Vertrauen in die Technik hingegen liefert die Forschung bereits Erkenntnisse, welche Determinanten die jeweiligen Dimensionen beeinflussen (Söllner et al. 2012b). Diese können als Grundlage ebenfalls für CloudComputing verwendet werden. Jedoch sollte im Falle des konkreten CloudComputing-Dienstes kritisch geprüft werden, ob diese für den Anwendungsfall relevant sind. Gemäß Söllner et al. (2012a) werden im Systemvertrauen die drei Vertrauensdimensionen von zwölf Determinanten beeinflusst. Für die Vertrauensdimension Performance wurden nach Söllner et al. (2012a) folgende Vertrauensdeterminanten identifiziert (vgl. Abbildung 2): 
- Eignung (umfasst die Einschätzung des Nutzers, wie gut das System grundsätzlich dazu geeignet ist, ihn bei der Erreichung des Ziels zu unterstützen),

- Informationsgenauigkeit (umfasst die Einschätzung des Nutzers, wie präzise die bereitgestellten Informationen sind),

- Verlässlichkeit (entspricht der Einschätzung des Nutzers, wie verlässlich das System über die Zeit hinweg ist) und

- Komplettheit (beschreibt die Einschätzung des Nutzers, dass das System sämtliche Funktionen beinhaltet, um das gewünschte Ziel zu erreichen). (Söllner et al. 2012a).

Die Vertrauensdimension Prozessnachvollziehbarkeit wird nach Söllner et al (2012a) von folgenden Vertrauensdeterminanten bestimmt (vgl. Abbildung 2):

- Konsistenz (beschreibt die Einschätzung des Nutzers, inwieweit sich das System konsistent, also widerspruchsfrei, verhält),

- Verständlichkeit (beinhaltet die Einschätzung des Nutzers, inwieweit für diesen verständlich ist, wie das System funktioniert),

- Kontrolle (bedeutet die Einschätzung des Nutzers bzgl. seiner wahrgenommenen Kontrolle über das System),

- Vorhersagbarkeit (umfasst die Einschätzung des Nutzers, inwieweit das zukünftige Verhalten des Systems antizipiert werden kann) (Söllner et al. 2012a).

Die dritte Vertrauensdimension für das Systemvertrauen, die Zweckklarheit, umfasst nach Söllner et al. (2012a) folgende Vertrauensdeterminanten (vgl. Abbildung 2):

- Kommunikation des Einsatzzweckes (beschreibt die Einschätzung des Nutzers, ob der gedachte Einsatzzweck des Systems von den Entwicklern kommuniziert wurde), 
- Wohlwollen der Entwickler (umfasst die Einschätzung des Nutzers, ob die Entwickler seine Interessen beachten) und

- Glaube (umschreibt die Einschätzung des Nutzers, dass dieser sich auch in Zukunft auf das System verlassen kann) (Söllner et al. 2012a).

Auf dieser theoretischen Grundlage werden nun im folgenden Schritt vertrauensbezogene Anforderungen abgeleitet, um im letzten Schritt exemplarisch Lösungen für die Vertrauensproblematiken im Cloud-Computing-Umfeld abzuleiten.

\subsection{Schritt 4: Ableitung vertrauensbezogener Anforderungen}

Im vierten Schritt folgt die Entwicklungsaktivität „Ableitung vertrauensbezogener Anforderungen“. Hierbei wird die Leitfrage beantwortet: „Welche Anforderungen können auf Basis der Determinanten abgeleitet werden?“.

Der Standard IEEE 610.12-1990 (IEEE 1990) definiert den Begriff $A n$ forderung (im englischen Original: requirement) wie folgt: „Eine Anforderung ist:

1) Eine Bedingung oder Eigenschaft, die ein System oder eine Person benötigt, um ein Problem zu lösen oder ein Ziel zu erreichen.

2) Eine Bedingung oder Eigenschaft, die ein System oder eine Systemkomponente aufweisen muss, um einen Vertrag zu erfüllen oder einem Standard, einer Spezifikation oder einem anderen formell aufgelegten Dokument zu genügen.

3) Eine dokumentierte Repräsentation einer Bedingung oder Eigenschaft wie in (1) oder (2) definiert." (Pohl 2007, S. 13).

Forschungsarbeiten liefern bereits eine Auswahl an vertrauensbezogenen Anforderungen für verschiedenste IT-Systeme (Hoffmann et al. 2012; Hoffmann/Söllner 2014; Hoffmann et al. 2013). Cloud-Computing-Anbieter, welche den Prozess zur systematischen Vertrauensunterstützung durchlaufen, können die nachfolgenden vertrauensbezogenen Anforderungen als Grundlage verwenden, sollten aber kritisch prüfen, ob diese für den konkreten An- 
wendungsfall relevant sind. Hoffmann (2014) identifizierte die folgenden vertrauensbezogenen Anforderungen, wobei diese dem Anbietervertrauen oder dem Systemvertrauen zugeordnet sind.

\section{Anforderungen zur Schaffung von Vertrauen in den Anbieter des Sys-} tems:

Nach Hoffmann (2014) soll der Anbieter ...

- Referenzen durch Dritte zur Applikation bereitstellen,

- die Bewertung der Applikation durch unabhängige Institutionen bereitstellen (Zertifizierung),

- Benutzern die Abgabe von Feedback zur Applikation ermöglichen und das Feedback von Benutzern zur Applikation veröffentlichen,

- den Benutzern den Kontakt zu ihm ermöglichen,

- eine Erklärung zum Datenschutz bereitstellen,

- eine Erklärung zur Datensicherheit bereitstellen (Hoffmann 2014).

\section{Anforderungen zur Schaffung von Vertrauen in das System:}

Nach Hoffmann (2014) soll der Anbieter ...

- verdeutlichen, welche Aufgaben die Applikation für die Benutzer erfüllt,

- Informationen über die Leistungsfähigkeit der Applikation bereitstellen,

- verdeutlichen, wie die Applikation ihre Aufgaben löst,

- die Benutzer darüber informieren, wie die Applikation funktioniert,

- eine Vorschau der Interaktion mit der Applikation bereitstellen (Hoffmann 2014).

Nach Hoffmann (2014) soll die Applikation ...

- die Benutzer bei Bedarf vor der Ausführung einer Funktion über diese Funktion informieren,

- den Benutzern den Status von Funktionen anzeigen, 
- die Benutzer bei Bedarf über durchgeführte Vorgänge in der Applikation informieren,

- die Benutzer bei Bedarf über Vorgänge informieren,

- den unberechtigten Zugriff auf gespeicherte Daten verhindern,

- Aktivierung und Deaktivierung von Funktionalitäten durch die Benutzer ermöglichen,

- den Benutzern die erfolgreiche Ausführung von Vorgängen bestätigen,

- Benutzern das Rückgängigmachen von Vorgängen ermöglichen,

- den Benutzern Eingaben bestätigen (Hoffmann 2014, S. 193-230).

Zudem kann die Vertrauenswürdigkeit des Anbieters und des Systems auch durch das Aufzeigen von Rechtskonformität bzw. Rechtsverträglichkeit gefördert werden (Jandt 2008; Hoffmann 2014). Dementsprechend kann Vertrauen auch durch eine rechtmäßige sowie rechtsverträgliche Gestaltung der Technik gefördert werden (Jandt 2008).

\subsection{Schritt 5: Ableitung vertrauensunterstützender Mehrwertdienste}

Im Rahmen der Entwicklungsaktivität „Ableitung vertrauensunterstützender Mehrwertdienste" wird die Leitfrage „Welche vertrauensunterstützenden Mehrwertdienste können auf Basis der Anforderungen abgeleitet werden?" beantwortet. Dazu werden anhand der vertrauensbezogenen Anforderungen konkrete Lösungen, Maßnahmen oder Gestaltungselemente abgeleitet, um so den Cloud-Computing-Dienst vertrauensförderlicher zu gestalten.

Unter Verwendung der individuellen Ergebnisse aus Schritt eins bis Schritt vier sollten Cloud-Computing-Anbieter befähigt sein, für den zu entwickelnden oder angebotenen Cloud-Dienst gezielt vertrauensunterstützende Mehrwertdienste abzuleiten und umzusetzen. Da die Anwendbarkeit und die Inhalte dieses Schrittes sehr stark von dem betrachteten Anwendungsfall abhängen, können keine allgemeingültigen vertrauensunterstützenden Mehr- 
wertdienste genannt werden. Dennoch werden im Folgenden beispielhaft einige Anregungen gegeben.

Um die Vertrauensdeterminante „Empfehlungen durch Dritte“ zu adressieren, sind folgende vertrauensunterstützenden Maßnahmen denkbar:

- Der Anbieter stellt eine Übersicht über die bisherigen Kunden bereit.

- Der Anbieter liefert einen Nachweis, dass auch unabhängige Institutionen den Dienst geprüft haben, z. B. durch den Erwerb und die präsente Darstellung eines Zertifizierungssiegels.

- Der Anbieter schafft Raum für Kundenrezensionen, bei dem bestehende Kunden ihre Erfahrungen mit dem Dienst und dem Anbieter potenziellen Neukunden zur Verfügung stellen.

Um die „Kundenorientierung“ stärker herauszustellen, können beispielhaft folgende Maßnahmen ergriffen werden:

- Der Anbieter betreibt eine 24-h-Hotline, um den (potenziellen) Kunden das Gefühl zu geben, im Notfall rund um die Uhr einen Ansprechpartner zu haben (Hoffmann 2014).

- Der Anbieter stellt dem Nutzer eine kostenlose Testversion zur Verfügung (Hoffmann 2014).

- Der Anbieter zeigt dem Kunden mit Hilfe einer Demonstration, welche Möglichkeiten zur Anpassung an die Kundenbedürfnisse bestehen.

Auch für die Vertrauensdeterminante „Kontrolle“ können anhand der Anforderungen verschiedenste vertrauensunterstützende Maßnahmen abgeleitet werden:

- Bei der Installation des Systems wird die Zustimmung vom Nutzer für alle Funktionen eingeholt, die kritisch für diesen sein könnten. Zudem sollte dieser die Möglichkeit haben, diese Zustimmung jederzeit zu verändern.

- Das System gibt dem Nutzer proaktiv Rückmeldung zum aktuellen Stand der durchgeführten Vorgänge (z.B. Vorgang erfolgreich beendet). 
- Der Anbieter stellt dem Kunden ohne dessen Aufforderung alle Informationen bereit, die dieser benötigt, um seinen rechtlichen Pflichten bei der Nutzung von Cloud-Computing-Diensten nachzukommen.

Zudem wurden weitere Maßnahmen zur vertrauensförderlichen Gestaltung von Cloud-Computing-Diensten mit Hilfe von Experteninterviews identifiziert (siehe Kapitel 21).

\subsection{Zusammenfassung}

In diesem Kapitel wurde die Methode zur Entwicklung vertrauensunterstützender Komponenten für sozio-technische ubiquitäre Systeme nach Söllner et al. (2012a) auf das Cloud-Computing-Umfeld übertragen und weiterentwickelt. Unter Anwendung des hier beschriebenen Prozesses zur systematischen Vertrauensunterstützung ist der Anbieter von Cloud-ComputingDiensten befähigt, diese zukünftig vertrauensförderlicher zu gestalten bzw. am Markt anzubieten. Die Abfolge der Schritte kann für jeden Cloud-Computing-Dienst durchlaufen werden. Dabei liefern die exemplarischen Antworten zu den Leitfragen bereits eine erste Hilfe zur Beantwortung. Dennoch sollten die Cloud-Computing-Anbieter die Übertragbarkeit dieser exemplarischen Anregungen auf den konkreten Anwendungsfall kritisch überprüfen.

\subsection{Literaturverzeichnis}

Buch, M.; Gebauer, L. (2013): Maßnahmenkatalog zur Vertrauenserhöhung im Cloud Computing. In: Leimeister, J.M. (Hrsg.), (4. Aufl.). Kassel, Deutschland: Working Paper Series, Chair for Information Systems, Kassel University.

Buch, M.; Gebauer, L.; Hoffmann, H. (2014): Vertrauen in Cloud Computing schaffen - Aber wie? In: WIRTSCHAFTSINFORMATIK \& MANAGEMENT, Vol. 03/2014 (2014), S. 67-77.

Hoffmann, A. (2014): Anforderungsmuster zur Spezifikation soziotechnischer Systeme - Standardisierte Anforderungen der Vertrauenswürdigkeit und Rechtsverträglichkeit (Dissertation) Kassel University Press, Kassel, Deutschland 2014.

Hoffmann, A.; Hoffmann, H.; Söllner, M. (2013): Fostering Initial Trust in Applications - Developing and Evaluating Requirement Patterns for Application Websites. Präsentiert auf der 21st European Conference on Information Systems, Utrecht, Niederlande. 
Hoffmann, A.; Söllner, M.; Hoffmann, H. (2012): Twenty software requirement patterns to specify recommender systems that users will trust. Präsentiert auf der 20th European Conference on Information Systems, Barcelona, Spanien.

Hoffmann, H.; Söllner, M. (2014): Incorporating behavioral trust theory into system development for ubiquitous applications. In: Personal and Ubiquitous Computing, Vol. 18 (2014) No. 1, S. 117-128.

IEEE (1990): Standard Glossary of Software Engineering Terminology, IEEE Std 610.12-1990. IEEE Computer Society Press 1990.

Jandt, S. (2008): Vertrauen im Mobile Commerce - Vorschläge für die rechtsverträgliche Gestaltung von Location Based Services, Nomos, Baden-Baden, Deutschland 2008.

Lee, J.D.; See, K.A. (2004): Trust in Automation: Designing for Appropriate Reliance. In: Human Factors: The Journal of the Human Factors and Ergonomics Society, Vol. 46 (2004) No. 1, S. 50-80.

Mayer, R.C.; Davis, J.H.; Schoorman, F.D. (1995): An Integrative Model of Organizational Trust. In: The Academy of Management Review, Vol. 20 (1995) No. 3, S. 709-734.

Pohl, K. (2007): Requirements Engineering: Grundlagen, Prinzipien, Techniken, Dpunkt-Verlag 2007.

Söllner, M.; Hoffmann, A.; Hoffmann, H.; Leimeister, J.M. (2012a): Vertrauensunterstützung für sozio-technische ubiquitäre Systeme. In: Zeitschrift für Betriebswirtschaft, (2012) No. 4, S. 109-140.

Söllner, M.; Hoffmann, A.; Hoffmann, H.; Wacker, A.; Leimeister, J.M. (2012b): Understanding the Formation of Trust in IT Artifacts. Präsentiert auf der 33rd International Conference on Information Systems, Orlando, USA.

Söllner, M.; Pavlou, P.; Leimeister, J.M. (2013): Understanding Trust in IT Artifacts - A new Conceptual Approach. Präsentiert auf dem 73th Academy of Management Annual Meeting, Orlando, USA. 